\title{
CityGuide: A Seamless Indoor-Outdoor Wayfinding System for People With Vision Impairments
}

\author{
Seyed Ali Cheraghi \\ sxcheraghi@shockers \\ .wichita.edu
}

\author{
Ali Almadan \\ aaalmadan@shockers \\ .wichita.edu
}

\author{
Vinod Namboodiri \\ vinod.namboodiri@ \\ wichita.edu
}

\author{
Wichita State University, Wichita, KS, USA
}

\begin{abstract}
GPS accuracy is poor in indoor environments and around buildings. Thus, reading and following signs still remains the most common mechanism for providing and receiving wayfinding information in such spaces. This puts individuals who are blind or visually impaired (BVI) at a great disadvantage. This work designs, implements, and evaluates a wayfinding system and smartphone application called CityGuide that can be used by BVI individuals to navigate their surroundings beyond what is possible with just a GPS-based system. CityGuide enables an individual to query and get turn-by-turn shortest route directions from an indoor location to an outdoor location. CityGuide leverages recently developed indoor wayfinding solutions in conjunction with GPS signals to provide a seamless indoor-outdoor navigation and wayfinding system that guides a BVI individual to their desired destination through the shortest route. Evaluations of CityGuide with BVI human subjects navigating between an indoor starting point to an outdoor destination within an unfamiliar university campus scenario showed it to be effective in reducing end-to-end navigation times and distances of almost all participants.
\end{abstract}

\section{Author Keywords}

Navigation and wayfinding; accessibility; vision impairments;

\section{CCS Concepts}

-Human-centered computing $\rightarrow$ Accessibility technologies;

\section{INTRODUCTION}

Wayfinding remains a challenge for people with disabilities in our communities. For outdoor environments, recent advances in satellite-based systems and mapping technologies along with the pervasiveness of smartphones provide an accurate and simple to use means for wayfinding. However, there remain many outdoor areas such as sidewalks, within and around office buildings, public recreational areas, and university campuses, where the effectiveness of satellite-based systems such as global positioning systems (GPS) is limited. This paper

Permission to make digital or hard copies of part or all of this work for personal or classroom use is granted without fee provided that copies are not made or distributed for profit or commercial advantage and that copies bear this notice and the full citation on the first page. Copyrights for third-party components of this work must be honored For all other uses, contact the Owner/Author.

ASSETS '19, October 28-30, 2019, Pittsburgh, PA, USA

(C) 2019 Copyright is held by the owner/author(s).

ACM ISBN 978-1-4503-6676-2/19/10.

https://doi.org/10.1145/3308561.3354621 proposes a wayfinding system and smartphone application called CityGuide that can be used by BVI individuals to navigate their surroundings beyond what is possible with just a GPS-based system. CityGuide enables an individual to query and get turn-by-turn shortest route directions from an indoor location to an outdoor location. When navigation starts within an indoor environment leading to or through any outdoor location, CityGuide leverages any Bluetooth-low energy (BLE) beacons in the indoor environments to guide the user to the best exit of the building that lies on the shortest path towards the eventual destination. Upon exiting an indoor environment, it seamlessly switches to utilize GPS signals towards the desired destination on the shortest route. CityGuide additionally implements mechanisms to make outdoor wayfinding more fine-grained and accurate to improve the navigation performance and experience of end-users.

\section{RELATED WORK}

There have been many recent efforts in indoor wayfinding utilizing wireless devices, such as radio-frequency identification or BLE, or computer vision to provide location information and context within such spaces $[8,4,7,5,6,4]$. While different technologies have been used for indoor wayfinding, outdoor wayfinding systems usually have relied on GPS technology for some or all their data gathering. GPS-based navigation apps such as Google Maps, Apple maps, Blindsquare [1], GetThere [2], and Microsoft Soundscape [3] provide routes in unfamiliar urban environments using different approaches. CityGuide combines a lot of the features of the above mentioned indoor and outdoor wayfinding systems/apps but adds the extra layer required to combine them and create a seamless indoor-outdoor navigation experience. It uses BLE beacons in the surrounding infrastructure to localize within indoor environments, utilizing pre-constructed maps from floor plans. This enables navigating indoor spaces effortlessly. In outdoor environments it utilizes GPS signals and pedestrian walking maps to provide turn-by-turn directions, combining information from any BLE beacons it encounters along the way (for example at entrances of other buildings along the way) and utilizing algorithms like dead reckoning to improve accuracy.

\section{SYSTEM OVERVIEW}

Upon activation, the CityGuide app on a smartphone detects the user's current location and waits for the user to provide the desired destination. The phrase from the user is then looked up in a database of points of interest (POIs) in the indoor space as well as sent as a query to the Google Places API for outdoor locations. If matches are found, they are listed out to 
the user one by one until the user confirms one of them. Upon confirmation that there is a match for the desired destination, CityGuide calculates the best available route from the user's location to one of the building's exit points (assuming the user is within a building and searches for a location outside the building) and subsequently to the destination in outdoor environments.

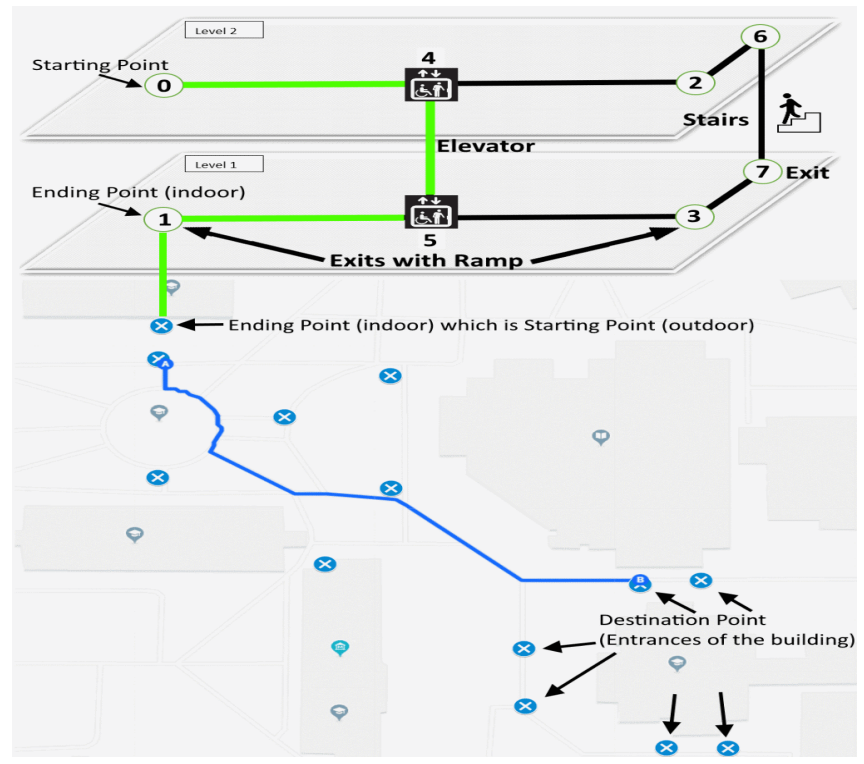

Figure 1: Jabara Hall floorplan modeled as a graph showing vertices and edges, beacon placement outside at the buildings entrances, and shortest routh from Jabara hall to the Wallace Hall.

\section{System Setup}

The current implementation of CityGuide utilizes methods previously developed in a BLE beacon-based indoor wayfinding system called GuideBeacon in [7]. Based upon that system's guidelines, beacons are affixed near each POI, and as users come in proximity of a beacon, a unique identifier is received from the beacon at the smartphone. Although placing and utilizing beacons in outdoor environments would increase the accuracy of outdoor navigation (due to GPS inaccuracies), this is cost-prohibitive and thus largely infeasible. However, there are some locations where beacons can be assumed to be present such as the entry/exit points of buildings, bus stops, and any major landmarks. Figure 1 shows beacon placement in Jabara Hall (beacons numbered from 0 to 7 ), beacon placement in outdoor environments (blue circles), and the experimental scenario that subjects used to evaluate the CityGuide. CityGuide was written as an app for the Android OS and can work using its native TalkBack accessibility tool. All tests were conducted on a Samsung Galaxy S7 phone that used a Wi-Fi connection to communicate to Google API to get outdoor routes as well as private servers.

\section{RESULTS}

Figure $2 \mathrm{a}$ and $2 \mathrm{~b}$ show the navigation time and distance required by each user tested with and some without the use of

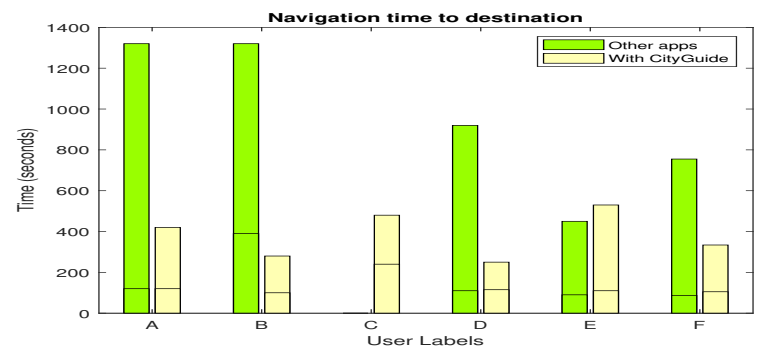

(a) Navigation time.

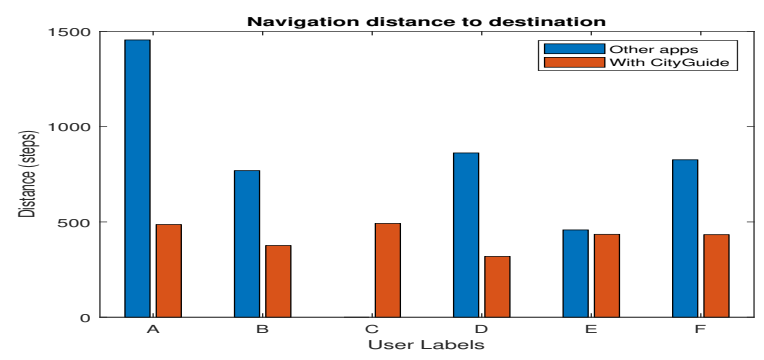

(b) Navigation distance.

Figure 2: Results comparing the use of other apps for indooroutdoor wayfinding to Cityguide for the navigaiton time and distance. The horizontal line in the middle of each result bar in (a) indicates the time to exit the building.

\begin{tabular}{|l|l|}
\hline User Label & Vision Category \\
\hline A & Blind, Cane user \\
\hline B & Only LP, Cane user \\
\hline C & Only LP, Cane user \\
\hline D & Blind, Guide Dog user \\
\hline E & Blind, Cane user \\
\hline F & Only LP, Cane user \\
\hline
\end{tabular}

Table 1: User information. LP is an abbreviation for light perception. All users except $\mathrm{C}$ (only used CityGuide) navigate to the destination without CityGuide and then with it.

CityGuide. In terms of the indoor part of the experiment without CityGuide (and with no others indoor wayfinding tools available), participants took varying amounts of time to exit the building, and (except user D) did not come out through the optimal exit towards the destination (Green route in figure 1). With CityGuide, they took similar times to come out, but all participants exited through the optimal exit which benefits the total time to navigate to the eventual destination. All BVI subjects took more time to complete the outdoor navigation part (and the entire start to destination exercise) except user E. It can be observed that for all users that used CityGuide, the steps taken are consistent (within a narrow range of about 100 steps) and less; on the other hand the steps taken by users not using CityGuide varied a lot, with some users not able to reach the destination.

\section{ACKNOWLEDGMENTS}

This paper was funded in part by the U.S. National Science Fundation (NSF) grant \#1737433. 


\section{REFERENCES}

[1] 2015. BlindSquare Indoor Navigation. (2015). http://blindsquare. com/indoor/

[2] 2019. GetThere GPS nav for blind. (2019). https://play.google.com/store/apps/details?id=com. LewLasher.getthere

[3] 2019. Microsoft Soundscape. https://www.microsoft.com/enus/research/product/soundscape/. (2019).

[4] 2019. Wayfindr Open Standard. (2019). https://www. wayfindr.net/

[5] Dragan Ahmetovic, Cole Gleason, Chengxiong Ruan, Kris Kitani, Hironobu Takagi, and Chieko Asakawa. 2016. NavCog: A Navigational Cognitive Assistant for the Blind. In Proceedings of the 18th International Conference on Human-Computer Interaction with Mobile Devices and Services (MobileHCI'16). ACM, New York, NY, USA, 90-99. DOI :

http://dx.doi.org/10.1145/2935334.2935361
[6] Dragan Ahmetovic, Masayuki Murata, Cole Gleason, Erin Brady, Hironobu Takagi, Kris Kitani, and Chieko Asakawa. 2017. Achieving Practical and Accurate Indoor Navigation for People with Visual Impairments. In Proceedings of the 14th Web for All Conference on The Future of Accessible Work (W4A '17). ACM, New York, NY, USA, Article 31, 10 pages. DOI : http://dx.doi.org/10.1145/3058555.3058560

[7] S. A. Cheraghi, V. Namboodiri, and L. Walker. 2016. Guidebeacon: Beacon-based indoor wayfinding for the blind, visually impaired, and disoriented. IEEE Pervasive Communications (PerCom) (2016).

[8] Jee-Eun Kim, Masahiro Bessho, Shinsuke Kobayashi, Noboru Koshizuka, and Ken Sakamura. 2016. Navigating Visually Impaired Travelers in a Large Train Station Using Smartphone and Bluetooth Low Energy. In Proceedings of the 31st Annual ACM Symposium on Applied Computing (SAC '16). 604-611. 